whose lower urinary tract symptoms benefited from neuromodulation were likely to report improved sexual functioning.

The authors assessed 121 patients (mean age 53.6 years) with overactive bladder $(n=83)$, chronic pelvic pain $(n=23)$ or nonobstructive urinary retention $(n=15)$ who received 12 weekly 30 min sessions of percutaneous tibialnerve stimulation. Sexual functioning was assessed before and after neuromodulatory therapy by means of a short questionnaire, completed by $88.4 \%$ and $86 \%$ of the patients before and after therapy, respectively.

About one-third of the cohort reported impaired baseline sexual functioning. The patients most likely to report improved sexual functioning (principally improved libido and increased frequency of sexual contact) after neuromodulatory therapy were females, patients with overactive bladder, and patients who requested continuation of therapy after the initial 12 weeks. The authors suggest that neuromodulation might decrease female patients' fears of incontinence (or actual incontinence) episodes during sexual activity.

The authors noted that 17 of 29 patients with overactive bladder who did not experience orgasm during sexual activity before neuromodulatory therapy did so afterwards; these findings might be attributable to the fact that micturition and sexual reflexes share common neural pathways.

Original article van Balken MR et al. (2006) Sexual functioning in patients with lower urinary tract dysfunction improves after percutaneous tibial nerve stimulation. Int $J$ Impot Res 18: 470-476

\section{Patients value quality of life over a survival advantage}

Research has shown that patients tend to choose aggressive treatments, even if there is little or no survival benefit. Most of these studies, however, investigated hypothetical situations; patients might decide differently when faced with a real-life choice. A team of Dutch researchers has examined actual patient treatment choices and the reasons behind them.

In the study, 150 patients with primary prostate cancer were offered a choice between two standard radiotherapy doses: $70 \mathrm{~Gy}$ and 74 Gy. Treatment options were discussed in an unbiased manner during a semistructured interview, with visual decision aids (pie charts). Patients who made a choice $(n=119)$ were asked to also indicate the relative importance given to factors such as survival, cure, and adverse effects.

Overall, $75 \%$ of patients chose the lower dose. Patient choices correlated strongly with their estimation of the importance of various factors: patients who chose the lower dose placed more importance on the risk of gastrointestinal and genitourinary problems, and patients who chose the higher dose placed more importance on survival and the risk of tumor recurrence. Exclusion of low-risk and elderly patients, who had the least to gain from aggressive treatment, reduced the proportion of patients who chose the lower dose to $61 \%$.

The authors conclude that patients' choices are based on reasonable assessments of their situations, and recommend that patients be included in the treatment decision-making process. They also note that patients are unlikely to welcome the current trend towards higher-dose radiotherapy until the risk of adverse effects declines.

Original article van Tol-Geerdink JJ et al. (2006) Do patients with localized prostate cancer really want more aggressive treatment? J Clin Oncol 24: 4581-4586

\section{Why is malpractice insurance so expensive?}

In the US, malpractice insurance premiums have risen by up to $57 \%$ over the last 3 years. Over half of urologists refer complex, difficult cases and limit the scope of their practice in an effort to avoid these costs. In light of the effect these strategies have on patient care, Perrotti et al. have investigated the factors involved in these premium rises.

They identified 469 consecutive malpractice claims settled with an indemnity payment from the records of an insurance company that insures over one-third of New York urologists. The claims were settled between 1985 and 2004, and totalled US\$99,335,341. Claims were most commonly related to postoperative events ( $n=101)$, negligent surgery $(n=96)$, failure to diagnose correctly $(n=60)$, administrative error $(n=21)$, and foreign bodies left after surgery $(n=20)$. No claims were related to laparoscopic or robotic-assisted procedures, but it is possible that such claims have not yet been resolved. 